\title{
A NOTE ON THE LOCAL INJECTIVITY OF WEAKLY DIFFERENTIABLE MAPPINGS HAVING CONSTANT JACOBIAN SIGN
}

\author{
MIHAI CRISTEA
}

\begin{abstract}
We give topological and analytical conditions in order that some weakly differentiable mappings with constant Jacobian sign to be locally injective, generalizing results from [10], [2], established for Sobolev mappings.
\end{abstract}

\section{Introduction}

The central result of this paper is the following theorem.

Theorem 1. Let $D \subset \mathrm{R}^{n}$ be open, $f: D \rightarrow \mathrm{R}^{n}$ continuous so that $f$ satisfies condition $(N)$ and suppose that $f$ has at a.e. point $x \in D$ a quasidifferential $L_{x}$ with $\operatorname{det} L_{x} \geq 0$. Then $f$ is weakly sense-preserving. If, in addition, $f$ is open, then $f$ is sense-preserving.

Throughout this paper we shall work with mappings $f: D \rightarrow \mathrm{R}^{n}$ defined on open sets from $\mathrm{R}^{n}$. Condition (N) requires that $f$ carries sets $A \subset D$ with $\mu_{n}(A)=0$ to sets with $\mu_{n}(f(A))=0$, where $\mu_{n}$ denotes the Lebesgue measure from $\mathrm{R}^{n}$. An $n \times n$ matrix $L$ is called a quasidifferential of $f$ at $x \in D$ if there exists $r_{i} \rightarrow 0$ so that for every $\varepsilon>0$, there is $i_{\varepsilon} \in \mathrm{N}$ so that

$$
\sup _{\|z-x\|=r_{i}}\|f(z)-f(x)-L(z-x)\| \leq \varepsilon \cdot r_{i}, \quad \text { for } \quad i \geq i_{\varepsilon} .
$$

A map $f$ can have different quasidifferentials at the same point. The concepts weakly "sense-preserving" and "sense-preserving" refer to the topological degree, see Section 2 for the definitions. We call a mapping $f$ open if it maps open sets to open sets. We wish to point out that the condition (N) assumption cannot be omitted even when $\operatorname{det} L_{x}>0$ a.e. and $f \in \bigcap_{p<n} W^{1, p}\left(D, \mathbf{R}^{n}\right)$, see [5]. On the other hand, if $f \in W^{1, n}\left(D, \mathrm{R}^{n}\right)$ and either $\operatorname{det} L_{x}>0$ a.e., $L_{x}=0$ a.e. in the set where $\operatorname{det} L_{x}=0$, or $f$ is open, then condition (N) is 
automatically satisfied, see [2], [6], [8]. For results related to Theorem 1, see Theorem 1.5 and Theorem 2.4 from [5]. See also [3] for some other results concerning the maps with finite distortion.

Theorem 1 together with its slightly more general version given in Section 3 allow us to prove extensions of certain results of Putten [10] and Fonseca and Gangbo [2] on the local invertibility of Sobolev mappings. For simplicity we only formulate here the following result and refer the reader to Section 2 and Section 3 for further conclusions.

Theorem 2. Let $D \subset \mathrm{R}^{n}$ be open, $f: D \rightarrow \mathrm{R}^{n}$ continuous, open and satisfying condition $(N)$, and suppose that $f$ has at a.e. point $x \in D$ a quasidifferential $L_{x}$ with $\operatorname{det} L_{x} \geq 0$. Then $B_{f} \subset \tilde{Z}_{f} \cup \tilde{S}_{f}$.

Here, $B_{f}$ consists of those points $x$ in $D$ for which $f$ fails to be a local homeomorphism at $x, \tilde{S}_{f}$ is the set of the points $x$ at which $f$ does not have a quasidifferential, $\tilde{Z}_{f}$ is the set of points where $f$ has only quasidifferentials $L$ with $\operatorname{det} L=0$.

\section{Proofs of the main results}

We begin with a Sard type lemma for quasidifferentiable maps.

Lemma 1. Let $D \subset \mathrm{R}^{n}$ be open and $f: D \rightarrow \mathrm{R}^{n}$ be continuous and open. Then $\mu_{n}\left(f\left(C_{f}\right)\right)=0$.

Here $C_{f}$ is the set of points where $f$ has a quasidifferential $L$ with $\operatorname{det} L=0$.

Proof. By usual covering arguments (see for instance [1]), it clearly sufficies to prove that, given $x \in C_{f}$, there is a sequence of radii $r_{i}$ tending to zero so that

$$
\lim _{i \rightarrow \infty} \frac{\mu_{n}\left(f\left(\bar{B}\left(x, r_{i}\right)\right)\right)}{\mu_{n}\left(B\left(x, r_{i}\right)\right)}=0 .
$$

For this, let $L$ be a quasidifferential with det $L=0$ and $\left(r_{i}\right)$ a corresponding sequence of radii. Because $L$ is not injective, there is an $(n-1)$-dimensional plane $T$ so that $f(x)+L(z-x) \in T$ for all $z \in \mathbf{R}^{n}$. Now, given $\varepsilon>0$,

$$
\|f(z)-f(x)-L(z-x)\| \leq \varepsilon r_{i}
$$

and

$\|f(z)-f(x)\| \leq\|f(z)-f(x)-L(z-x)\|+\|L(z-x)\| \leq(1+\|L\|) r_{i}$,

when $i$ is sufficiently large and $\|z-x\|=r_{i}$. Thus $f\left(S\left(x, r_{i}\right)\right)$ is contained in a product type set $U$ obtained from an $(n-1)$-ball of radius no more than $(1+\|L\|) r_{i}$ in $T$ and an interval of lenght $2 \varepsilon r_{i}$. 
As $f$ is open and continuous, also $f\left(B\left(x, r_{i}\right)\right) \subset U$. The claim follows because $\varepsilon$ was arbitrary.

The topological degree is widely used in the proofs, and we use the notations from [7] and [2]. If $D \subset \mathrm{R}^{n}$ is open, bounded, $f: \bar{D} \rightarrow \mathrm{R}^{n}$ is continuous and $p \notin f(\partial D)$, we denote by $d(f, D, p)$ the topological degree of $f$, on $D$, at the point $p$. We say that $f: D \rightarrow \mathrm{R}^{n}$ is weakly sense-preserving (sensepreserving) if $d(f, Q, y) \geq 0 \quad(d(f, Q, y)>0)$ for every domain $Q$ with $\bar{Q} \subset D$.

Proof of Theorem 1. Let $U \subset \subset D$ be open and $x_{0} \in U$ such that $f\left(x_{0}\right) \notin$ $f(\partial U)$. Pick an open ball $W$ with $f\left(x_{0}\right) \in W$ and so that $\bar{W} \cap f(\partial U)=\emptyset$. We shall associate to each point $x \in U \backslash \tilde{S}_{f}$ a sequence of balls $B\left(x, r_{i}\right)$ shrinking to $x$ and corresponding open sets $V_{i}$ with $f\left(S\left(x, r_{i}\right)\right) \subset V_{i}$ as follows.

If $x \in \tilde{Z}_{f}$, we use the same argument as in the proof of Lemma 1 to select a sequence $\left(r_{i}\right)$ tending to zero and open sets $V_{i}$ with $f\left(\bar{B}\left(x, r_{i}\right)\right) \subset V_{i}$ and so that

$$
\frac{\mu_{n}\left(V_{i}\right)}{\mu_{n}\left(B\left(x, r_{i}\right)\right)} \leq \frac{\mu_{n}(W)}{2 \cdot \mu_{n}(U)} .
$$

Then $d\left(f, B\left(x, r_{i}\right), y\right)=0$ for every $y \notin V_{i}$.

Given $x \in U \backslash\left(\tilde{Z}_{f} \cup \tilde{S}_{f}\right)$, let $L_{x}$ be a quasidifferential of $f$ at $x$ with $\operatorname{det} L_{x} \neq 0$. Let $\left(r_{i}\right)$ be a sequence as in the definition of quasidifferentiability, corresponding to $L_{x}$.

We can find as in [12], pages 325-334, a sequence of numbers $\lambda_{i}>0$ tending to zero, so that

$$
f\left(S\left(x, r_{i}\right)\right) \subset V_{i}:=f(x)+\left(L_{x}\left(B\left(0,\left(1+\lambda_{i}\right) r_{i}\right)\right) \backslash L_{x}\left(B\left(0,\left(1-\lambda_{i}\right) r_{i}\right)\right)\right)
$$

with

$$
d\left(f, B\left(x, r_{i}\right), y\right)=0 \quad \text { if } \quad y \notin f(x)+L_{x}\left(B\left(0,\left(1+\lambda_{i}\right) r_{i}\right)\right),
$$

and

$$
d\left(f, B\left(x, r_{i}\right), y\right)=1 \quad \text { if } \quad y \in f(x)+L_{x}\left(B\left(0,\left(1-\lambda_{i}\right) r_{i}\right)\right)
$$

and so again

$$
\frac{\mu_{n}\left(V_{i}\right)}{\mu_{n}\left(B\left(x, r_{i}\right)\right)} \leq \frac{\mu_{n}(W)}{2 \mu_{n}(U)} .
$$

Our balls in this case will be the balls $B\left(x, r_{i}\right)$. 
By the Vitali covering theorem (see [9], page 26), we can select pairwise disjoint balls $B_{j}$ from the above two collections of balls so that

$$
\mu_{n}\left(\left(U \backslash \tilde{S}_{f}\right) \backslash \bigcup_{1}^{\infty} B_{j}\right)=0 .
$$

Let $K=U \backslash \bigcup_{1}^{\infty} B_{j}$. Then $\mu_{n}(K)=0$, and hence $\mu_{n}(f(K))=0$, as $f$ satisfies condition (N). Consider the sets $V_{j}$ associated to the balls $B_{j}$. From the definition of the sets $V_{j}$ we notice that

$$
d\left(f, B\left(x, r_{j}\right), y\right) \geq 0
$$

whenever $y \notin V_{j}$. Moreover,

$$
\sum_{1}^{\infty} \mu_{n}\left(V_{j}\right) \leq \frac{\mu_{n}(W)}{2 \mu_{n}(U)} \cdot \sum_{1}^{\infty} \mu_{n}\left(B_{j}\right) \leq \frac{\mu_{n}(W)}{2} .
$$

Thus there is at least one point $y$ with

$$
y \in W \backslash\left(f(K) \cup \bigcup_{1}^{\infty} V_{j}\right) .
$$

Notice that $f^{-1}(y) \cap U \subset \bigcup_{1}^{\infty} B_{j}$. Let $I=\left\{j \in \mathbf{N} \mid f^{-1}(y) \cap B_{j} \neq \emptyset\right\}$. Then $I$ is finite and we obtain

$$
d\left(f, U, f\left(x_{0}\right)\right)=d(f, U, y)=d\left(f, \bigcup_{j \in I} B_{j}, y\right)=\sum_{j \in I} d\left(f, B_{j}, y\right) \geq 0 .
$$

It follows that $f$ is weakly sense-preserving.

Suppose then that $f$ is open. By Lemma 1 , it follows that $\mu_{n}\left(f\left(\tilde{Z}_{f}\right)\right)=0$. Let again $x_{0}, U$, and $W$ be as in the first part of the proof. Let $B_{0}$ be an open ball centered at $x_{0}$ so that $f\left(B_{0}\right) \subset W$. Because $f$ is open and $\mu_{n}\left(f\left(\tilde{Z}_{f} \cup \tilde{S}_{f}\right)\right)=0$, there is a point $x \in B_{0}$ with $f(x) \in W \backslash\left(f\left(\tilde{Z}_{f} \cup \tilde{S}_{f}\right)\right)$. We can find a radius $r>0$ so that $\bar{B}(x, r) \subset B_{0}, f(x) \notin f(S(x, r))$ and $d(f, B(x, r), f(x))=1$. By the first part of the proof, we conclude that

$$
\begin{aligned}
d\left(f, U, f\left(x_{0}\right)\right) & =d(f, U, f(x)) \\
& =d(f, U \backslash \bar{B}(x, r), f(x))+d(f, B(x, r), f(x)) \geq 1,
\end{aligned}
$$

as desired.

Our next result deals with local invertibility. 
THeorem 3. Let $D \subset \mathrm{R}^{n}$ be open, $f: D \rightarrow \mathrm{R}^{n}$ continuous, nonsingular and satisfying condition $(N)$ and $\mu_{n}\left(f\left(\tilde{Z}_{f}\right)\right)=0$. Suppose that $f$ has at a.e. point $x \in D$ a quasidifferential $L_{x}$ at $x$ with $\operatorname{det} L_{x} \geq 0$. Then, for every $x \notin \tilde{Z}_{f} \cup \tilde{S}_{f}$, there is $r_{x}>0$ and a neighborhood $Q_{x}$ of $x$ so that

$$
f \mid Q_{x} \backslash\left(\tilde{Z}_{f} \cup \tilde{S}_{f}\right): Q_{x} \backslash\left(\tilde{Z}_{f} \cup \tilde{S}_{f}\right) \rightarrow B\left(f(x), r_{x}\right) \backslash f\left(\left(\tilde{Z}_{f} \cup \tilde{S}_{f}\right) \cap Q_{x}\right)
$$

is a homeomorphism and for every $y \in B\left(f(x), r_{x}\right), f^{-1}(y) \cap Q_{x}$ is connected.

Here, a map $f: D \rightarrow \mathbf{R}^{n}$ is nonsingular if int $f(U) \neq \emptyset$ for every open nonempty set $U \subset D$. We denote by $N(y, f, A)$ the number of elements of the set $A \cap f^{-1}(y)$.

Proof of Theorem 3. Since $f$ is nonsingular and $\mu_{n}\left(f\left(\tilde{Z}_{f} \cup \tilde{S}_{f}\right)\right)=0$, we see that int $f^{-1}\left(f\left(\tilde{Z}_{f} \cup \tilde{S}_{f}\right)\right)=\emptyset$, hence $\operatorname{int}\left(\tilde{Z}_{f} \cup \tilde{S}_{f}\right)=\emptyset$. Let $x \notin$ $\tilde{Z}_{f} \cup \tilde{S}_{f}$. Then $f$ has a quasidifferential $L_{x}$ at $x$ with $\operatorname{det} L_{x}>0$, and we find $\delta_{x}>0$ so that $f(x) \notin f\left(S\left(x, \delta_{x}\right)\right)$ and $d\left(f, B\left(x, \delta_{x}\right), f(x)\right)=1$. Let $r_{x}>0$ be so that $\bar{B}\left(f(x), r_{x}\right) \cap f\left(S\left(x, \delta_{x}\right)\right)=\emptyset$ and let $Q_{x}$ be the component of $f^{-1}\left(B\left(f(x), r_{x}\right)\right)$ containing $x$. Then $Q_{x} \subset B\left(x, \delta_{x}\right), Q_{x}$ is a neighbourhood of $x$ and

$$
d\left(f, B\left(x, \delta_{x}\right), y\right)=d\left(f, B\left(x, \delta_{x}\right), f(x)\right)=1 \text { for every } y \in B\left(f(x), r_{x}\right),
$$

and hence $B\left(f(x), r_{x}\right) \subset f\left(B\left(x, \delta_{x}\right)\right)$. Let $y \in B\left(f(x), r_{x}\right) \backslash f\left(\tilde{Z}_{f} \cup \tilde{S}_{f}\right)$ and suppose that there exist $x_{1}, x_{2} \in B\left(x, \delta_{x}\right), x_{1} \neq x_{2}$ so that $f\left(x_{1}\right)=f\left(x_{2}\right)=y$. Then $x_{1}, x_{2} \notin \tilde{Z}_{f} \cup \tilde{S}_{f}$ and we can find $r_{1}, r_{2}>0$ so that

$$
\bar{B}\left(x_{1}, r_{1}\right) \cup \bar{B}\left(x_{2}, r_{2}\right) \subset B\left(x, \delta_{x}\right), \quad \bar{B}\left(x_{1}, r_{1}\right) \cap \bar{B}\left(x_{2}, r_{2}\right)=\emptyset,
$$

$y \notin f\left(S\left(x_{1}, r_{1}\right) \cup S\left(x_{2}, r_{2}\right)\right) \quad$ and $\quad d\left(f, B\left(x_{1}, r_{1}\right), y\right)=d\left(f, B\left(x_{2}, r_{2}\right), y\right)=1$.

From Theorem 1 we see that $f$ is weakly sense-preserving; hence

$$
d\left(f, B\left(x, \delta_{x}\right) \backslash\left(\bar{B}\left(x_{1}, r_{1}\right) \cup \bar{B}\left(x_{2}, r_{2}\right)\right), y\right) \geq 0,
$$

and we obtain that

$$
\begin{aligned}
1=d\left(f, B\left(x, \delta_{x}\right), y\right)= & d\left(f, B\left(x, \delta_{x}\right) \backslash\left(\bar{B}\left(x_{1}, r_{1}\right) \cup \bar{B}\left(x_{2}, r_{2}\right)\right), y\right) \\
& +d\left(f, B\left(x_{1}, r_{1}\right), y\right)+d\left(f, B\left(x_{2}, r_{2}\right), y\right) \geq 2
\end{aligned}
$$

which represents a contradiction. We proved that $f$ is injective on

$$
\left(B\left(x, \delta_{x}\right) \cap f^{-1}\left(B\left(f(x), r_{x}\right)\right)\right) \backslash f^{-1}\left(f\left(\tilde{Z}_{f} \cup \tilde{S}_{f}\right)\right),
$$

and hence $f$ is injective on $Q_{x} \backslash f^{-1}\left(f\left(\tilde{Z}_{f} \cup \tilde{S}_{f}\right)\right)$. 
Let now $z \in Q_{x}$ be so that $f$ is open at $z$ and suppose that there is $w \neq$ $z, w \in B\left(x, \delta_{x}\right)$ so that $f(z)=f(w)$. We can find $U_{1}, U_{2}$ open, disjoint so that $z \in U_{1}, w \in U_{2}, \bar{U}_{1} \cup \bar{U}_{2} \subset B\left(x, \delta_{x}\right)$, and using the openness of $f$ at $z$ and the continuity of $f$ at $w$, we can assume that

$$
f\left(U_{2}\right) \subset f\left(U_{1}\right) \subset B\left(f(x), r_{x}\right) .
$$

Since int $f^{-1}\left(f\left(\tilde{Z}_{f} \cup \tilde{S}_{f}\right)\right)=\emptyset$, we can find a point $a \in U_{2} \backslash f^{-1}\left(f\left(\tilde{Z}_{f} \cup \tilde{S}_{f}\right)\right)$, hence we can find a point $b \in U_{1} \backslash f^{-1}\left(f\left(\tilde{Z}_{f} \cup \tilde{S}_{f}\right)\right)$ so that $f(a)=f(b)$, which represents a contradiction, since we proved that $f$ is injective on

$$
B\left(x, \delta_{x}\right) \cap f^{-1}\left(B\left(f(x), r_{x}\right) \backslash f\left(\tilde{Z}_{f} \cup \tilde{S}_{f}\right)\right) .
$$

We proved that

$$
N\left(f(z), f, B\left(x, \delta_{x}\right)\right)=1 \quad \text { if } \quad z \in Q_{x} \quad \text { and } \quad f \text { is open at } z .
$$

Since $f$ is open at every point $z \in Q_{x} \backslash\left(\tilde{Z}_{f} \cup \tilde{S}_{f}\right)$, it results that

$$
f^{-1}(f(z)) \cap B\left(x, \delta_{x}\right)=\{z\} \quad \text { for every } \quad z \in Q_{x} \backslash\left(\tilde{Z}_{f} \cup \tilde{S}_{f}\right) .
$$

We see that $f$ is injective on $Q_{x} \backslash\left(\tilde{Z}_{f} \cup \tilde{S}_{f}\right)$,

$$
\begin{aligned}
Q_{x} \cap\left(\tilde{Z}_{f} \cup \tilde{S}_{f}\right) & =Q_{x} \cap f^{-1} f\left(Q_{x} \cap\left(\tilde{Z}_{f} \cup \tilde{S}_{f}\right)\right), Q_{x} \backslash\left(\tilde{Z}_{f} \cup \tilde{S}_{f}\right) \\
& =Q_{x} \cap f^{-1}\left(f\left(Q_{x} \backslash\left(\tilde{Z}_{f} \cup \tilde{S}_{f}\right)\right)\right),
\end{aligned}
$$

and

$$
f \mid Q_{x} \backslash\left(\tilde{Z}_{f} \cup \tilde{S}_{f}\right): Q_{x} \backslash\left(\tilde{Z}_{f} \cup \tilde{S}_{f}\right) \rightarrow B\left(f(x), r_{x}\right) \backslash f\left(Q_{x} \cap\left(\tilde{Z}_{f} \cup \tilde{S}_{f}\right)\right)
$$

is a homeomorphism.

Now, let $y \in B\left(f(x), r_{x}\right)$ and let $A_{1}, A_{2}$ be two different components of $f^{-1}(y) \cap B\left(x, \delta_{x}\right)$. Then $A_{1}$ and $A_{2}$ are compact and $A_{1} \cup A_{2} \subset B\left(x, \delta_{x}\right)$.

From Theorem 1, $f$ is sense-preserving, hence it is quasiopen (Lemma 5.5, page 147 in [11]). Let $Q_{1}, Q_{2}$ be open so that $A_{1} \subset Q_{1}, A_{2} \subset Q_{2}$ and $\bar{Q}_{1} \cup \bar{Q}_{2} \subset B\left(z, \delta_{x}\right)$. Since $f\left(A_{1}\right)=f\left(A_{2}\right)=\{y\}$ and $f$ is quasiopen, we can find a point $w \in f\left(Q_{1}\right) \cap f\left(Q_{2}\right) \cap B\left(f(x), r_{x}\right)$, and since $f$ is sensepreserving, we have that

$d\left(f, Q_{1}, w\right) \geq 1, \quad d\left(f, Q_{2}, w\right) \geq 1, \quad d\left(f, B\left(x, \delta_{x}\right) \backslash\left(\bar{Q}_{1} \cup \bar{Q}_{2}\right), w\right) \geq 0$.

We obtain that

$$
\begin{aligned}
1 & =d\left(f, B\left(x, \delta_{x}\right), f(x)\right)=d\left(f, B\left(x, \delta_{x}\right), w\right) \\
& =d\left(f, B\left(x, \delta_{x}\right) \backslash\left(\bar{Q}_{1} \cup \bar{Q}_{2}\right), w\right)+d\left(f, Q_{1}, w\right)+d\left(f, Q_{2}, w\right) \geq 2,
\end{aligned}
$$


which represents a contradiction.

We therefore proved that $f^{-1}(y) \cap B\left(f(x), r_{x}\right)$ is connected for every $y \in$ $B\left(f(x), r_{x}\right)$.

Proof OF Theorem 2. We apply (1) and Lemma 1 to see that $B_{f} \subset \tilde{Z}_{f} \cup \tilde{S}_{f}$.

\section{Further results}

An $n \times n$ matrix $L$ is called the approximate differential of $f$ at $x$ if the maps $f_{h}: B(0,1) \rightarrow \mathrm{R}^{n}$, defined by $f_{h}(y)=\frac{f(x+h y)-f(x)}{h}$ for $y \in B(0,1)$ and $h>0$, converge to $L$ in measure on $B(0,1)$. Then $L$ is unique, and such a matrix $L$ is called the weak differential of $f$ at $x$ if in addition $L$ is a quasidifferential of $f$ at $x$. We also say that $f$ is weakly differentiable at $x$ and we denote $J_{f}(x)=\operatorname{det} L$. We put $Z_{f}=\{x \in D \mid f$ is weakly differentiable at $x$ and $\left.J_{f}(x)=0\right\}$ and $S_{f}=\{x \in D \mid f$ is not weakly differentiable at $x\}$. The version of Theorem 1 for weakly differentiable mappings is:

Theorem 4. Let $D \subset \mathrm{R}^{n}$ be open, $f: D \rightarrow \mathrm{R}^{n}$ continuous, nonsingular and satisfying condition $(N)$, weakly differentiable a.e. so that $J_{f}(x) \geq 0$ a.e. in $D$. Then $f$ is sense-preserving.

PRoof. We see from Theorem 1 that $f$ is weakly sense-preserving. We remark that in the proof of the sense-preserveness of the map $f$ from Theorem 1, we used the openness of $f$ only to state that $f$ is nonsingular and that $\mu_{n}\left(f\left(\tilde{Z}_{f}\right)\right)=0$. Since we see from [2], Theorem 5.6, page 110, that $\mu_{n}\left(f\left(Z_{f}\right)\right)=0$, without the openness assumption, we argue as is Theorem 1 to find that $f$ is sense-preserving.

Using Sard's lemma for weakly differentiable mappings from [2], Theorem 5.6, page 110, instead of our version of Sard's lemma from Lemma 1, and replacing the set $\tilde{Z}_{f}$ by $Z_{f}$ and the set $\tilde{S}_{f}$ by $S_{f}$, we can formulate Theorem 2 and Theorem 3 in this setting. We give only the version of Theorem 3.

Theorem 5. Let $D \subset \mathrm{R}^{n}$ be open, $f: D \rightarrow \mathrm{R}^{n}$ continuous, open, satisfying condition $(N)$, weakly differentiable a.e., so that $J_{f}(x) \geq 0$, a.e. in $D$. Then $B_{f} \subset Z_{f} \cup S_{f}$.

We formulate now some simple conditions for nonsingularity, which can be used to obtain new versions of the theorems where it appears this property. First, a map $f: D \rightarrow \mathbf{R}^{n}$ is called a light map if $\operatorname{dim} f^{-1}(y) \leq 0$ for every $y \in \mathbf{R}^{n}$. We know from [4], page 92, that every continuous, light map is nonsingular. Also, if $f: D \rightarrow \mathrm{R}^{n}$ is continuous, so that $\operatorname{int}\left(\tilde{Z}_{f} \cup \tilde{S}_{f}\right)=\emptyset$ or $\operatorname{int}\left(Z_{f} \cup S_{f}\right)=\emptyset$, then $f$ is nonsingular.

ACKNOWLEDGEMENTS. I wish to thank to P. Koskela for his comments. The author is supported by the Research Grant 6154, Project A10/2000 of CNCSIZ. 


\title{
REFERENCES
}

1. Cristea, M., Some remarks about Sard's theorem and the condition (N) satisfied by the inverse map $f^{-1}$, An. Univ. Bucuresti Mat. 41, 1 (1992), 23-28.

2. Fonseca, I., and Gangbo, W., Degree Theory in Analysis and Appl., Oxford Univ. Press, 1995.

3. Heinonen, J., and Koskela, P., Sobolev mappings with integrable dilatation, Arch. Rational Mech. Anal. 125 (1993), 81-97.

4. Hurewicz, W., and Walman, H., Dimension Theory, Princeton Univ. Press, 1941.

5. Kauhanen, J., Koskela, P., and Malý, J., Mappings of finite distortion: discreteness and openness, Arch. Rational Mech. Anal. 160 (2001), 135-151.

6. Kauhanen, J., Koskela, P., and Malý, J., Mappings offinite distortion: Condition(N), Michigan Math. J. 49 (2001), 169-181.

7. Lloyd, N. G., Degree Theory, Cambridge Univ. Press, Cambridge, 1978.

8. Malý, J., and Martio, O., Lusin's condition $(N)$ and mappings of the class $W^{1, n}$, J. Reine Angew. Math. 458 (1995), 19-36.

9. Mattila, P., Geometry of Sets and Measures in Euclidean Spaces, Cambridge Univ. Press, 1995.

10. Putten, R., A note on the local invertibility of Sobolev functions, Math. Scand. 83 (1998), 255-264.

11. Rickman, S., Quasiregular mappings, Ergeb. Math. Grenzgeb. (3) 26, 1993.

12. Radó, T., and Reichelderfer, P. V., Continuous transformations in analysis, Springer-Verlag, Berlin, 1955.

\author{
UNIVERSITY OF BUCHAREST \\ FACULTY OF MATHEMATICS \\ STR. ACADEMIEI 14, R-70109 \\ BUCHAREST \\ ROMANIA \\ E-mail: cristea@geometry.math.unibuc.ro
}

